\title{
Evanescent character of the repulsive thermal Casimir force
}

\author{
Vitaly B. Svetovoy \\ MESA + Research Institute, University of Twente, P.O. Box 217, 7500 AE Enschede, The Netherlands
}

(Received 30 June 2007; revised manuscript received 21 August 2007; published 5 December 2007)

\begin{abstract}
The physical origin of the negative thermal correction to the Casimir force between metals is clarified. For this purpose the asymptotic behavior of the thermal Casimir force is analyzed at large and small distances in the real frequency representation. Contributions from propagating and evanescent waves are considered separately. At large distances they cancel each other in substantial degree so that only the attractive Lifshitz limit survives. At smaller separations the repulsive evanescent contribution of $s$-polarization dominates in the case of two metals or a metal and a high-permittivity dielectric. Common origin and order of magnitude of the repulsion in these two cases demonstrate naturalness of the controversial large thermal correction between metals.
\end{abstract}

DOI: 10.1103/PhysRevA.76.062102

PACS number(s): $12.20 . \mathrm{Ds}, 42.50 . \mathrm{Lc}, 42.50 . \mathrm{Pq}$

Over the last decade there was a growing interest in the Casimir force [1] that was measured in a series of recent experiments [2]. Particularly the thermal part of the force was a subject of active and often controversial discussion (see [3] for a review). Here we concentrate on the thermal force, which does not include the zero point fluctuations. The repulsion discussed in this paper has the meaning of a negative thermal correction to the force at zero temperature, but the total force is always attractive [4]. At large distances $a$ $\gg \hbar c / T\left(k_{B}=1\right)$, the thermal force is given by the Lifshitz limit [4-6]

$$
F_{\text {Lif }}=\frac{T}{16 \pi a^{3}} \int_{0}^{\infty} d x x^{2}\left[\frac{\left(\varepsilon_{1}+1\right)\left(\varepsilon_{2}+1\right)}{\left(\varepsilon_{1}-1\right)\left(\varepsilon_{2}-1\right)} e^{x}-1\right]^{-1},
$$

where $a$ is the distance between parallel plates and $T$ is the temperature of the system. This formula was derived for two dielectric plates with the static dielectric constants $\varepsilon_{1}$ and $\varepsilon_{2}$. The force between two ideal metals can be found from Eq. (1) as the limit $\varepsilon_{1,2} \rightarrow \infty$ that gives $F_{L i f}=T \zeta(3) / 8 \pi a^{3}$. This equation became one of the points of controversy $[7,8]$ because direct calculation of the thermal force between ideal metals [9] gave the result, which is two times larger. This contradiction found its resolution $[10,11]$. At large distances only low frequency electromagnetic (EM) fluctuations contribute to the force. In this limit the $s$-polarized EM field degenerates to a pure magnetic one, which penetrates freely via a nonmagnetic metal [11]. On the contrary, the $p$ polarized field is pure electric and reflected by the metal. For the ideal metal both polarizations are reflected and the force will be two times larger. In this sense the ideal metal is rather the limit case of a superconductor than of a normal metal [12].

The difference between ideal and real metals manifests itself also at small distances $a \ll \hbar c / T$. The force between ideal metals is attractive and small [9]. On the contrary, the force between real metals is relatively large and repulsive [7]. This difference did not yet find a clear physical explanation.

In this paper it is demonstrated that at distances $a$ $\lesssim \hbar c / T$ the evanescent contribution of $s$-polarization to the thermal Casimir force dominates for two metals or in the case of a metal and a high-permittivity dielectric. For both material configurations the force is repulsive but in the latter case it is free of controversies accompanying the interaction of metals. For ideal metals the evanescent contribution vanishes but propagating one is naturally small.

Due to technical convenience, in most cases the force is calculated using the Lifshitz formula [5] written via imaginary frequencies [4,6]. However, in this form significant physical information is lost. Originally this formula was presented in the real frequency domain [6] where the force is naturally composed of two contributions of the fluctuating fields: propagating waves (PW) and evanescent waves (EW). It is well known from optics that each component can be manipulated independently. In the case of the Casimir force the PW and EW components respond differently on the change of the material or variation of the distance. Understanding of this behavior is crucial for tailoring the force.

Recently, Intravaia and Lamrecht [13] demonstrated that at zero temperature, $T=0$, propagating modes give an attractive contribution to the force between metals while evanescent modes are responsible for the repulsion. For $p$ polarization both contributions are large but cancel each other in substantial degree so that the resulting force is attractive and small. One might hope to shift the balance to the repulsion using nanostructured materials [13]. It is not clear for the moment how realistic this suggestion is, but in this paper it is shown that the repulsion can dominate at least for the thermal part of the force.

The thermal force also revealed interesting cancellations between EW and PW contributions. At large distances significant cancellations are realized for dielectrics [12] and metals [14]. The cancellation is reduced in the nonequilibrium situation when the temperatures of the bodies are different [12]. It has to be stressed that in the situation out of equilibrium the real frequency representation is the only one possible due to the absence of analyticity [12].

If the wave vector in the vacuum gap is $\mathbf{k}=\left(q_{x}, q_{y}, k_{0}\right)$ with $q=\sqrt{q_{x}^{2}+q_{y}^{2}}$ and $k_{0}=\sqrt{\omega^{2} / c^{2}-q^{2}}$, then in the real frequency domain the thermal force is $[6,12] F=F_{P W}+F_{E W}$, where 


$$
\begin{aligned}
F_{P W}(a, T)= & -\frac{\hbar}{8 \pi^{2} a^{3}} \operatorname{Re} \int_{0}^{\infty} \frac{d \omega}{e^{\hbar \omega / T}-1} \int_{0}^{\omega / \omega_{c}} d y y^{2} \\
& \times \sum_{\mu=s, p} \frac{R^{\mu}(\omega, y) e^{i y}}{1-R^{\mu}(\omega, y) e^{i y}}, \\
F_{E W}(a, T)= & \frac{\hbar}{8 \pi^{2} a^{3}} \operatorname{Im} \int_{0}^{\infty} \frac{d \omega}{e^{\hbar \omega / T}-1} \int_{0}^{\infty} d y y^{2} \\
& \times \sum_{\mu=s, p} \frac{R^{\mu}(\omega, i y) e^{-y}}{1-R^{\mu}(\omega, y) e^{-y}} .
\end{aligned}
$$

Here $R^{\mu}=r_{1}^{\mu} r_{2}^{\mu}$, where $r_{m}^{\mu}(m=1,2)$ is the Fresnel reflection coefficient for body 1 or 2 and transverse electric $(\mu=s)$ or magnetic $(\mu=p)$ polarization. The integration variable $y$ is expressed via the physical values as $y=2 a k_{0}$ for PW and $y$ $=2 a\left|k_{0}\right|$ for EW. The distance dependence is included in the characteristic frequency $\omega_{c}=c / 2 a$. In terms of $\omega$ and $y$ the reflection coefficients are

$$
r_{m}^{s}=\frac{y-s_{m}}{y+s_{m}}, \quad r_{m}^{p}=\frac{\varepsilon_{m} y-s_{m}}{\varepsilon_{m} y+s_{m}},
$$

where $s_{m}=\sqrt{\omega^{2}\left(\varepsilon_{m}-1\right) / \omega_{c}^{2}+y^{2}}$ and $\varepsilon_{m}=\varepsilon_{m}(\omega)$ is the dielectric function of the body $m$.

Consider first the case of two similar metals, which can be described by the Drude dielectric function $\varepsilon(\omega)=1$ $-\omega_{p}^{2} / \omega\left(\omega+i \omega_{\tau}\right)$, where $\omega_{p}$ and $\omega_{\tau}$ are the plasma and relaxation frequencies, respectively. For the qualitative analysis it will be assumed that $\omega_{p} \rightarrow \infty, \omega_{\tau} \rightarrow 0$ (but both finite). Actual values of the parameters will be taken into account in numerical calculations.

At large distances, $a \gg \hbar c / T \equiv \lambda_{T}$ (thermal wavelength), PW contribution can be found [12] using first the multiplereflection expansion $R e^{i y} /\left(1-R e^{i y}\right)=\sum_{n=1}^{\infty} R^{n} e^{i n y}$ to avoid poles and after that one can safely put $R=1$ for both polarizations. The result will be

$$
F_{P W}(a, T)=\frac{T}{8 \pi a^{3}} \zeta(3)(1+1),
$$

where we separated two equal contributions from $s$ and $p$ polarizations. Of course, this result coincides with the force between ideal metals and one could expect that the EW contribution must be zero. But this is not true.

For $s$-polarized EW the reflection coefficient is

$$
r^{s}(\omega, i y)=\frac{y-\sqrt{\frac{\omega_{p}^{2}}{\omega_{c}^{2}} \frac{\omega}{\omega+i \omega_{\tau}}+y^{2}}}{y+\sqrt{\frac{\omega_{p}^{2}}{\omega_{c}^{2}} \frac{\omega}{\omega+i \omega_{\tau}}+y^{2}}} .
$$

If we neglect $y$ in Eq. (6) in comparison with $\omega_{p} / \omega_{c}$, then $r^{s}$ will be real and the EW contribution will vanish. The nonzero result for $F_{E W}^{s}$ originates from very low frequencies

$$
\omega \lesssim \omega_{\tau}\left(\frac{\omega_{c}}{\omega_{p}}\right)^{2} .
$$

At these frequencies one can expand $e^{\hbar \omega / T}-1 \approx \hbar \omega / T$ in Eq. (3). The resulting integral does not depend on the frequency scale. It means that even arbitrarily small $\omega_{\tau}$ plays a role in keeping the EW contribution finite [14] as follows:

$$
F_{E W}^{s}(a, T)=-\frac{T}{8 \pi a^{3}} \zeta(3) .
$$

As a result the PW and EW contributions precisely cancel each other for $s$ polarization as it happens for dielectrics. It must be so because a nonmagnetic metal is transparent for $s$ polarization in the low frequency limit as was explained above.

For $p$ polarization very low frequencies (7) are not important because $r^{p}$ has a significant imaginary part up to $\omega$ $\sim \omega_{\tau}$. The reflection coefficient is not scale invariant and the result of integration in Eq. (3) will depend on $\omega_{\tau}$. It is straightforward to show that $F_{E W}^{p} \sim \omega_{\tau}$ for $\hbar \omega_{\tau} \ll T$ or $F_{E W}^{p}$ $\sim \omega_{\tau}^{1 / 2}$ in the opposite limit. In our qualitative approximation we have to take $F_{E W}^{p}=0$. Therefore, for $p$ polarization there is no cancellation between PW and EW contributions and we recover the result (1).

It is interesting to see how the result will change at smaller distances. If the distance is small in comparison with the thermal wavelength but large in comparison with the penetration depth, $c / \omega_{p} \ll a \ll \lambda_{T}$, then the EW contribution will not change at all. This is because for $s$ polarization the low frequencies (7) still dominate and the conclusion on the behavior of $F_{E W}^{p}$ also remains true. In contrast, the PW contribution changes significantly. At small distances there is no room for standing waves between the plates and the only pressure that is important is the pressure from the back sides of the plates due to the blackbody radiation.

$$
F_{P W}(a, T)=\frac{\pi^{2}}{90} \frac{T^{4}}{\hbar^{3} c^{3}}(1+1) .
$$

This force is very small and we come to the conclusion that at these distances the dominant component of the total force is $s$-polarized EW as follows:

$$
F(a, T) \simeq-\frac{T}{8 \pi a^{3}} \zeta(3) .
$$

This force is repulsive and pure evanescent. To the best of our knowledge, evanescent character of the thermal force at small distances is stressed here, although one specific example was demonstrated numerically [15] and rejected as unacceptable.

Of course, Eq. (10) gives only the leading term and corrections due to finite $\omega_{p}$ and $\omega_{\tau}$ are important for realistic materials but qualitatively the result is not changed. It is demonstrated in Fig. 1, which shows different components of the force in Fig. 1(a) found by the numerical integration of Eqs. (2) and (3) at $T=300 \mathrm{~K}$ with the Drude parameters of $\mathrm{Au}: \omega_{p}=9.0 \mathrm{eV}, \omega_{\tau}=0.035 \mathrm{eV}$. The total force and total contributions from PW and EW are shown in Fig. 1(b). All components of the force are normalized to the natural value 


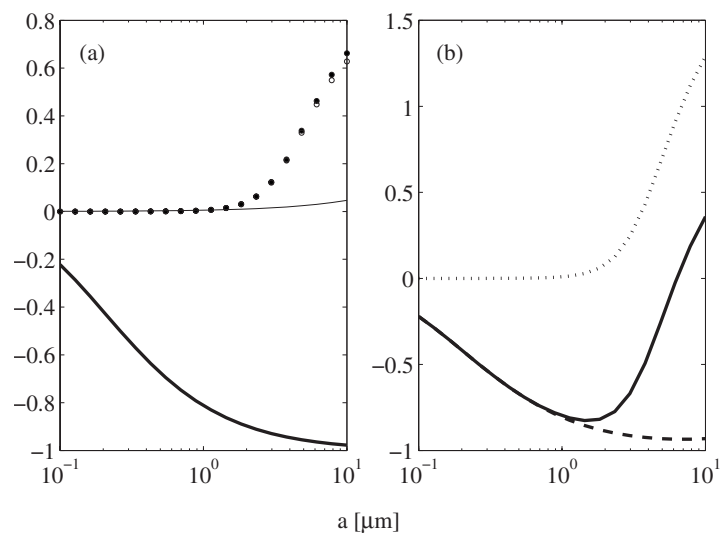

FIG. 1. (a) Distance dependence for different components of the thermal force between metals normalized to $T \zeta(3) / 8 \pi a^{3}$. Solid circles correspond to $F_{P W}^{S}$, open circles correspond to $F_{P W}^{p}$, the thick line corresponds to $F_{E W}^{s}$, and the thin line corresponds to $F_{E W}^{p}$. (b) Distance behavior of the total force (solid line), total PW (dotted), and EW (dashed) contributions.

$T \zeta(3) / 8 \pi a^{3}$. To get the result with respect to the force at $T$ $=0, \pi^{2} \hbar c / 240 a^{4}$, one has to multiply all the curves to the factor $1.163 a / \lambda_{T}$.

The repulsive thermal force between metals was discovered by Boström and Sernelius [7]. This conclusion was disputed [16] on the basis that the Lifshitz formula could not be applicable to metals and the Fresnel reflection coefficients (4) have to be modified at low frequencies. Interaction between a dielectric and a metal is free of controversies because $r_{1}^{s} r_{2}^{s}$ is safely going to zero in the low frequency limit even if the metal is ideal. But in this case a weak repulsive force also was demonstrated [17]. Below it is shown that the repulsion in the system metal dielectric also originates from the evanescent $s$-polarized waves. Moreover, for highpermittivity dielectrics the repulsion can be of the same order of magnitude as for metals.

Dielectrics with high permittivity are discussed as the materials for capacitors and resonators [18] in the $\mathrm{GHz}$ region. The dielectric function for some of them shows no absorption up to a few THz. For qualitative analysis it will be assumed that in the range of important frequencies, $\omega$ $\lesssim T / \hbar$, the dielectric can be described by a constant high permittivity $\varepsilon_{2} \gg 1$. Frequency dependent dielectric functions of both metal and dielectric will be used for numerical calculations.

Very low frequencies (7) do not play a significant role in the case of metal and dielectric and for the qualitative analysis we can take the reflection coefficients of the metal as $r_{1}^{s}$ $=-1$ and $r_{1}^{p}=+1$. Then the asymptotic behavior of the force at large and small distances can be found analytically.

At large distances the force is not sensitive to the materials at all. This is because grazing waves are important in this limit [12]. For these waves any material is close to a perfect reflector. For $s$ polarization $R^{s} \rightarrow+1$ as for two metals and we reproduce the result for that configuration as follows:

$$
F_{P W}^{s}=\frac{T}{8 \pi a^{3}} \zeta(3), \quad F_{E W}^{s}=-\frac{T}{8 \pi a^{3}} \zeta(3)
$$

But in contrast with the metals this result is true at much shorter distances $a \gg \lambda_{T} \varepsilon_{2}^{-1 / 2}$, where $r_{2}^{s} \approx-1$ is a good approximation. The total contribution of $s$ polarization is zero as expected. For $p$ polarization $r_{2}^{p} \approx 1$ at the distance $a$ $\gg \lambda_{T} \varepsilon_{2}^{1 / 2}$, which is much larger than $\lambda_{T}$. Then

$$
F_{P W}^{p}=-\frac{3}{4} \frac{T}{8 \pi a^{3}} \zeta(3), \quad F_{E W}^{p}=\frac{7}{4} \frac{T}{8 \pi a^{3}} \zeta(3) .
$$

The factor $-3 / 4$ in $F_{P W}^{p}$ originates from the fact that $R^{p} \rightarrow$ -1 in contrast with the case of $s$ polarization. This is a specific property of the metal-dielectric configuration. For both metal-metal and dielectric-dielectric configurations $R^{p} \rightarrow+1$. The total contribution from $p$ polarization survives and coincides with the Lifshitz limit (1) for $\varepsilon_{1} \rightarrow \infty$ and $\varepsilon_{2} \gg 1$. It is interesting to note that the repulsive character of $\mathrm{EW}$ is not a universal property as Eq. (12) demonstrates.

The small distance limit is realized for $s$ polarization at $a \ll \lambda_{T} \varepsilon_{2}^{-1 / 2}$. In this limit it was found

$$
F_{P W}^{s}=-\frac{\pi^{2} T^{4}}{90 \hbar^{3} c^{3}} \frac{3 \sqrt{\varepsilon_{2}}}{2}, \quad F_{E W}^{s}=-\frac{\pi^{2} T^{4}}{90 \hbar^{3} c^{3}} \varepsilon_{2}^{3 / 2} .
$$

Both contributions are repulsive and large in comparison with the blackbody pressure, but the EW contribution dominates at $\varepsilon_{2} \gg 1$. For $p$ polarization one can separate the range of very small distances $a \ll \lambda_{T} \varepsilon_{2}^{-3 / 2}$, but it has no particular interest because at large $\varepsilon_{2}$ it becomes shorter than the penetration depth for the metal, $c / \omega_{p}$. Instead, we present the result in the intermediate range $\lambda_{T} \varepsilon_{2}^{-3 / 2} \ll a \ll \lambda_{T} \varepsilon_{2}^{-1 / 2}$,

$$
F_{P W}^{p}=-\frac{\pi^{2} T^{4}}{90 \hbar^{3} c^{3}} \frac{3 \sqrt{\varepsilon_{2}}}{4}, \quad F_{E W}^{p}=\frac{T^{2} \ln \left(\varepsilon_{2}^{3 / 2} a / \lambda_{T}\right)}{24 a^{2} \hbar c \sqrt{\varepsilon_{2}}} .
$$

Comparing EW components in Eqs. (13) and (14) in the distance range $\lambda_{T} \varepsilon_{2}^{-3 / 2} \ll a \ll \lambda_{T} \varepsilon_{2}^{-1 / 2}$ one can see that $F_{E W}^{p}$ $\ll\left|F_{E W}^{s}\right|$. We can conclude that at small distances the EW contribution from an $s$-polarized field is the largest in the absolute value and repulsive. When the distance becomes larger, $\left|F_{E W}^{s}\right|$ increases approaching the limit (11). $F_{E W}^{p}$ also increases but stays much smaller than $\left|F_{E W}^{s}\right|$ because the former one reaches the asymptotic value (12) only at very large distances $a \gg \lambda_{T} \varepsilon_{2}^{1 / 2}$. When the distance becomes larger than $\lambda_{T} \varepsilon_{2}^{-1 / 2}$ the PW component in Eq. (11) becomes important and $F_{E W}^{s}$ is not dominant anymore. It means that the total force has to have a minimum where it is repulsive.

Figure 2 shows the numerical results for different components of the thermal force. The calculations were done for the ideal metal and dielectric with the frequency independent permittivity $\varepsilon_{2}=100$ at $T=300 \mathrm{~K}$. Figure 2(a) shows behavior at large distances. An important result of the numerical analysis is that the large distance limit is realized at $a$ approximately 100 times larger than $\lambda_{T} \varepsilon_{2}^{-1 / 2}$ for $s$ polarization or $\lambda_{T} \varepsilon_{2}^{1 / 2}$ for $p$ polarization. As one can see for $p$ polarization the PW and EW components did not fully reach the asymptotic values $-3 / 4$ and $7 / 4$ even at $a=1000 \mu \mathrm{m}$. Nonmonotonic behavior of $F_{P W}^{p}$ also should be mentioned. At 


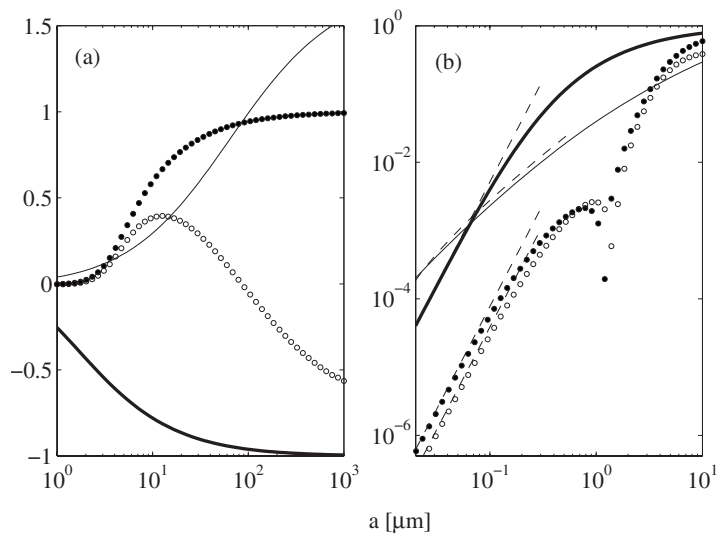

FIG. 2. Different components of the thermal force between metal and dielectric. Notations are similar to that in Fig. 1(a). Panel (a) shows large distance behavior. Panel (b) shows small distance behavior for absolute values of the forces. The dashed lines correspond to the asymptotics in Eqs. (13) and (14).

smaller distances the absolute values of the PW and EW components are shown in Fig. 2(b). The dashed lines represent the asymptotics (13) and (14).

Figure 3(a) shows the total PW and EW contributions together with the total force. It demonstrates the expected minimum of the total force. Figure 3(b) shows the total force between $\mathrm{SrTiO}_{3}$ single crystal [18] and Au. Frequency dependence of the dielectric functions of both materials was taken into account. The results are presented for three different temperatures. Influence of the temperature is significant because the absorption resonances of $\mathrm{SrTiO}_{3}$ are somewhat below room temperature. Frequency dependence of $\varepsilon_{2}$ in-

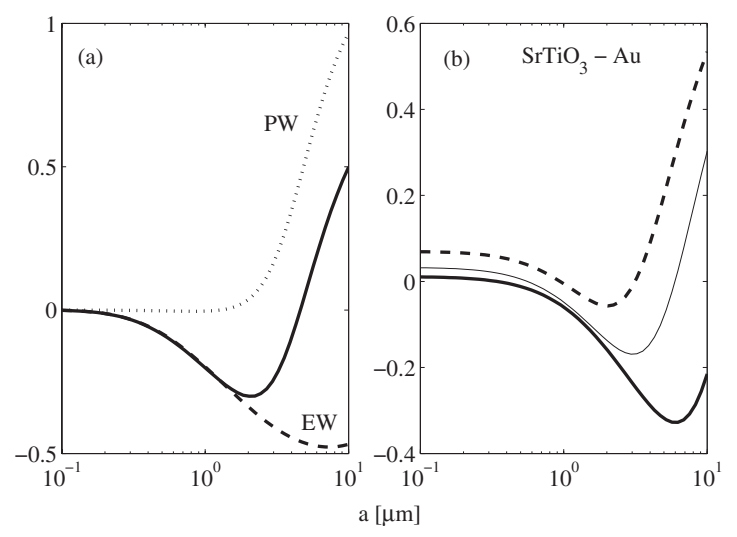

FIG. 3. (a) Total PW (dotted), total EW (dashed), and the total force (solid) for the ideal metal and ideal dielectric with $\varepsilon_{2}=100$. (b) The total force between realistic materials: $\mathrm{Au}$ and $\mathrm{SrTiO}_{3}$, $\varepsilon_{2}(0)=320$. Dashed, thin solid, and thick solid lines correspond to $T=300,200$, and $100 \mathrm{~K}$, respectively.

creases both EW components, thus reducing the repulsive force. Of course, with the temperature decrease the minimum becomes deeper and its position is shifted to larger distances.

In conclusion, we analyzed the PW and EW contributions to the thermal Casimir force between two metals and a metal and a high-permittivity dielectric. For both material configurations the repulsive $s$-polarized EW contribution dominates at $a \leqq \lambda_{T}$. The thermal repulsion between metals was disputed in the literature but the result of this paper demonstrates that a similar effect is realized for an uncontroversial case of metal and dielectric.
[1] H. B. G. Casimir, Proc. K. Ned. Akad. Wet. 51, 793 (1948).

[2] S. K. Lamoreaux, Phys. Rev. Lett. 78, 5 (1997); B. W. Harris, F. Chen, and U. Mohideen, Phys. Rev. A 62, 052109 (2000); T. Ederth, ibid. 62, 062104 (2000); H. B. Chan, V. A. Aksyuk, R. N. Kleiman, D. J. Bishop, and F. Capasso, Science 291, 1941 (2001); G. Bressi, G. Carugno, R. Onofrio, and G. Ruoso, Phys. Rev. Lett. 88, 041804 (2002); R. S. Decca, D. López, E. Fischbach, and D. E. Krause, ibid. 91, 050402 (2003).

[3] K. A. Milton, J. Phys. A 37, R209 (2004).

[4] I. E. Dzyaloshinskii, E. M. Lifshitz, and L. P. Pitaevskii, Adv. Phys. 10, 165 (1961).

[5] E. M. Lifshitz, Dokl. Akad. Nauk SSSR 100, 879 (1955).

[6] L. D. Landau and E. M. Lifshitz, Electrodynamics of Continuous Media (Pergamon Press, Oxford, 1963).

[7] M. Boström and B. E. Sernelius, Phys. Rev. Lett. 84, 4757 (2000).

[8] M. Bordag, B. Geyer, G. L. Klimchitskaya, and V. M. Mostepanenko, Phys. Rev. Lett. 85, 503 (2000); V. B. Svetovoy and M. V. Lokhanin, Phys. Lett. A 280, 177 (2001).

[9] J. Mehra, Physica 37, 145 (1967); L. S. Brown and G. J.
Maclay, Phys. Rev. 184, 1272 (1969).

[10] J. S. Høye, I. Brevik, J. B. Aarseth, and K. A. Milton, Phys. Rev. E 67, 056116 (2003); M. Boström and B. E. Sernelius, Physica A 339, 53 (2004).

[11] V. B. Svetovoy and R. Esquivel, Phys. Rev. E 72, 036113 (2005).

[12] M. Antezza, L. P. Pitaevskii, S. Stringari, and V. B. Svetovoy, Phys. Rev. Lett. 97, 223203 (2006); e-print arXiv:0706.1850, Phys. Rev. A (to be published).

[13] F. Intravaia and A. Lambrecht, Phys. Rev. Lett. 94, 110404 (2005).

[14] V. B. Svetovoy and R. Esquivel, J. Phys. A 39, 6777 (2006).

[15] J. R. Torgerson and S. K. Lamoreaux, Phys. Rev. E 70, 047102 (2004).

[16] B. Geyer, G. L. Klimchitskaya, and V. M. Mostepanenko, Phys. Rev. A 67, 062102 (2003).

[17] B. Geyer, G. L. Klimchitskaya, and V. M. Mostepanenko, Phys. Rev. A 72, 022111 (2005).

[18] T. Tsurumi, T. Teranishi, S. Wada, H. Kakemoto, O. Fukunaga, M. Nakada, and J. Akedo, J. Ceram. Soc. Jpn. 114, 774 (2006). 